SECTION 4. Computer science, computer engineering and automation.

Erik Nurlanovich Bayandiyev senior lecturer, Department of Mathematics, Taraz State University named after M.Kh.Dulaty, Kazakhstan erikn87@mail.ru

Lyazat Rysbekovna Seytbekova Associate professor, candidate of physical and mathematical sciences, Department of «Mathematics and MTM», Taraz State Pedagogical Institute, Kazakhstan

Aynur Tursynkhanovna Tolkynbayeva senior lecturer, Department of Mathematics, Taraz State University named after M.Kh.Dulaty, Kazakhstan

\title{
ON SOME ALGORITHMS FOR THE NUMERICAL SOLUTION OF THE CAUCHY PROBLEM FOR A SYSTEM OF DIFFERENTIAL EQUATIONS OF THE FIRST ORDER RUNGE - KUTT METHOD IN DELPHI
}

Abstract: This article discusses some aspects of application and computer realization of the numerical solution of differential equations on Delphi with use of widespread RungeKutt method.

Key words: Cauchy problem, Runge-Kutt method, algorithm, Delphi, program.

\section{О НЕКОТОРЫХ АЛГОРИТМАХ ЧИСЛЕННОГО РЕШЕНИЯ ЗАДАЧИ КОШИ ДЛЯ СИСТЕМЫ ДИФФЕРЕНЦИАЛЬНЫХ УРАВНЕНИЙ ПЕРВОГО ПОРЯДКА МЕТОДОМ РУНГЕ - КУТТА НА ЯЗЫКЕ DЕLРНI}

Аннотация: В данной статье рассматриваются некоторые моменты применения и компьютерной реализачии численного решения дифференциальных уравнений на Дельфи с применением широко распространенного метода Рунге-Кутта.

Ключевые слова: задача Кочи, метод Рунге-Кутта, алгоритм, дельфи, программа.

Пусть дана система двух дифференциальных уравнений первого порядка:

$$
\left\{\begin{array}{l}
y_{1}^{\prime}=f_{1}\left(x, y_{1}, y_{2}\right) \\
y_{2}^{\prime}=f_{2}\left(x, y_{1}, y_{2}\right)
\end{array}\right.
$$

Решением системы (1) называется пара функций $\varphi_{1}(x)$ и $\varphi_{2}(x)$, при подстановке которых в систему получаются тождества:

$$
\varphi_{1}^{\prime}=f_{1}\left(x, \varphi_{1}(x), \varphi_{2}(x)\right), \varphi_{2}^{\prime}=f_{2}\left(x, \varphi_{1}(x), \varphi_{2}(x)\right) .
$$

Решению

$$
\left\{\begin{array}{l}
\mathrm{y}_{1}=\varphi_{1}(x) \\
\mathrm{y}_{2}=\varphi_{2}(x)
\end{array}\right.
$$

системы уравненний (1) соответствует интегральная кривая в пространсте трех измерений $\left(x, y_{1}, y_{2}\right)$. Задача Коши для системы состоит в нахождении решения системы (1), удовлетворяющего начальным условиям [1-5]

$$
\left.\mathrm{y}_{1}\right|_{x=x_{0}}=y_{10},\left.\mathrm{y}_{2}\right|_{x=x_{0}}=y_{20} \text {. }
$$

Постановка задачи Коши для системы $\mathrm{n}$ дифференциальных уравнений первого порядка аналогична задаче (1-2), а именно: требуется найти решение системы 
при начальных начальных условиях

$$
\left\{\begin{array}{c}
y_{1}^{\prime}=f_{1}\left(x, y_{1}, y_{2}, \ldots, y_{n}\right) \\
y_{2}^{\prime}=f_{2}\left(x, y_{1}, y_{2}, \ldots, y_{n}\right) \\
\ldots \ldots \ldots \ldots \ldots \ldots \ldots \ldots \ldots \ldots \ldots \ldots \\
y_{n}^{\prime}=f_{n}\left(x, y_{1}, y_{2}, \ldots, y_{n}\right)
\end{array}\right.
$$

$$
\left.\mathrm{y}_{1}\right|_{x=x_{0}}=y_{10},\left.\mathrm{y}_{2}\right|_{x=x_{0}}=y_{20}, \ldots,\left.\mathrm{y}_{n}\right|_{x=x_{0}}=y_{n 0} \text {. }
$$

Теорема существования и единственности решения задачи Коши (3-4) имеет формулировку, аналогичную приведенной для частного случая $n=2$.

Если ввести векторные обозначения

$$
y(x)=\left(\begin{array}{c}
y_{1}(x) \\
y_{2}(x) \\
\ldots \\
y_{n}(x)
\end{array}\right), y^{\prime}(x)=\left(\begin{array}{c}
y_{1}^{\prime}(x) \\
y_{2}^{\prime}(x) \\
\ldots \\
y_{n}^{\prime}(x)
\end{array}\right), f(x, y)=\left(\begin{array}{c}
f_{1}(x, y) \\
f_{2}(x, y) \\
\ldots \\
f_{n}(x, y)
\end{array}\right), y_{0}=\left(\begin{array}{c}
y_{10} \\
y_{20} \\
\ldots \\
y_{n 0}
\end{array}\right) .
$$

то задача Коши (3-4) в векторной форме запишется так:

$$
y^{\prime}=f(x, y),\left.\quad y\right|_{x=x_{0}}=y_{0}
$$

Численное решение задачи Коши (5) состоит в том, что на сетке отрезка [a,b] требуется получить приближенные значения координат вектора $y(x)$ в узлах сетки $x_{i}$ $(i=1,2, \ldots, m)$.

Обозначим вектор, аппроксимирующий решение, через

$$
y_{i} \approx y\left(x_{i}\right)(i=1,2, \ldots, m)
$$

а его координаты - через

$$
y_{k i}(k=1,2, \ldots, n ; i=1,2, \ldots, m) \text { так, что } y_{k i} \approx y\left(x_{k i}\right)
$$

или

$$
y_{i}=\left(\begin{array}{c}
y_{1 i} \\
y_{2 i} \\
\cdots \\
y_{n i}
\end{array}\right) \approx y\left(x_{i}\right)=\left(\begin{array}{c}
y_{1}\left(x_{i}\right) \\
y_{2}\left(x_{i}\right) \\
. \\
y_{n}\left(x_{i}\right)
\end{array}\right) \quad(i=1,2, \ldots, m) .
$$

Будем искать решение на равномерной сетке с шагом $h=\frac{(b-a)}{m}$.

Величина погрешности численного метода оценивается величиной $d=$ $\max _{1 \leq i \leq m}\left\{d_{i}\right\}$, где $d_{i}$ - погрешность решения на сетке с шагом $h$ в точке $x_{i}$ :

$$
d_{i}(h)=\max _{1 \leq k \leq n}\left\{\left|y_{k i}(h)-y_{k}\left(x_{i}\right)\right|\right\}
$$

Практически погрешность в точке $x_{i}$ оценивается по формуле Рунге, аналогичной

$$
\left|\varphi\left(x_{i}\right)-y_{i}\left(\frac{h}{2}\right)\right| \approx \frac{\left|y_{i}(h)-y_{i}\left(\frac{h}{2}\right)\right|}{2^{p}-1} .
$$

А именно , пусть

$$
y_{i}(h)=\left(\begin{array}{c}
y_{1 i}(h) \\
y_{2 i}(h) \\
. \\
y_{n i}(h)
\end{array}\right), \quad y_{i}\left(\frac{h}{2}\right)=\left(\begin{array}{c}
y_{1 i}\left(\frac{h}{2}\right) \\
y_{2 i}\left(\frac{h}{2}\right) \\
\cdot \\
y_{n i}\left(\frac{h}{2}\right)
\end{array}\right)
$$

-значения численного решения в точке $x_{i}$, полученные для шагов $h$ и $\frac{h}{2}$ соответственно; тогда погрешность $d_{i}$ в точке $x_{i}$ для вычисления с шагов $\frac{h}{2}$ выражается приближенным равенством

$$
d_{i}\left(\frac{h}{2}\right) \approx \frac{\max _{1 \leq k \leq n}\left\{\left|y_{k i}(h)-y_{k}\left(x_{i}\right)\right|\right\}}{2^{p}-1}
$$


где $p$ - порядок точности численного метода.

Пример. Найти численное решение задачи Коши для системы двух дифференциальных уравнений на языке Delphi

$$
\begin{gathered}
\left\{\begin{array}{c}
y_{1}^{\prime}=y_{2}, \\
y_{2}^{\prime}=-y_{1} ;
\end{array}\right. \\
\left.\mathrm{y}_{1}\right|_{x=x_{0}}=y_{10},\left.\mathrm{y}_{2}\right|_{x=x_{0}}=y_{20} .
\end{gathered}
$$

Задача Коши для дифференциального уравнения второго порядка

$$
y^{\prime \prime}+y=0,\left.\quad y\right|_{x=x_{0}}=y_{0},\left.\quad y^{\prime}\right|_{x=x_{0}}=y_{0}^{\prime}
$$

приводится к задаче Коши для предыдущей системы, если обозначить

$$
y_{1}(x)=y(x), y_{2}=y_{1}^{\prime}(x) \text { и } y_{10}=y_{0}, y_{20}=y_{0}^{\prime} .
$$

Вычисления правых частей дифференциальных уравнений

$$
\begin{gathered}
f_{1}\left(x, y_{1}, y_{2}\right)=y_{2} \\
f_{2}\left(x, y_{1}, y_{2}\right)=-y_{1}
\end{gathered}
$$

ведется в программе:

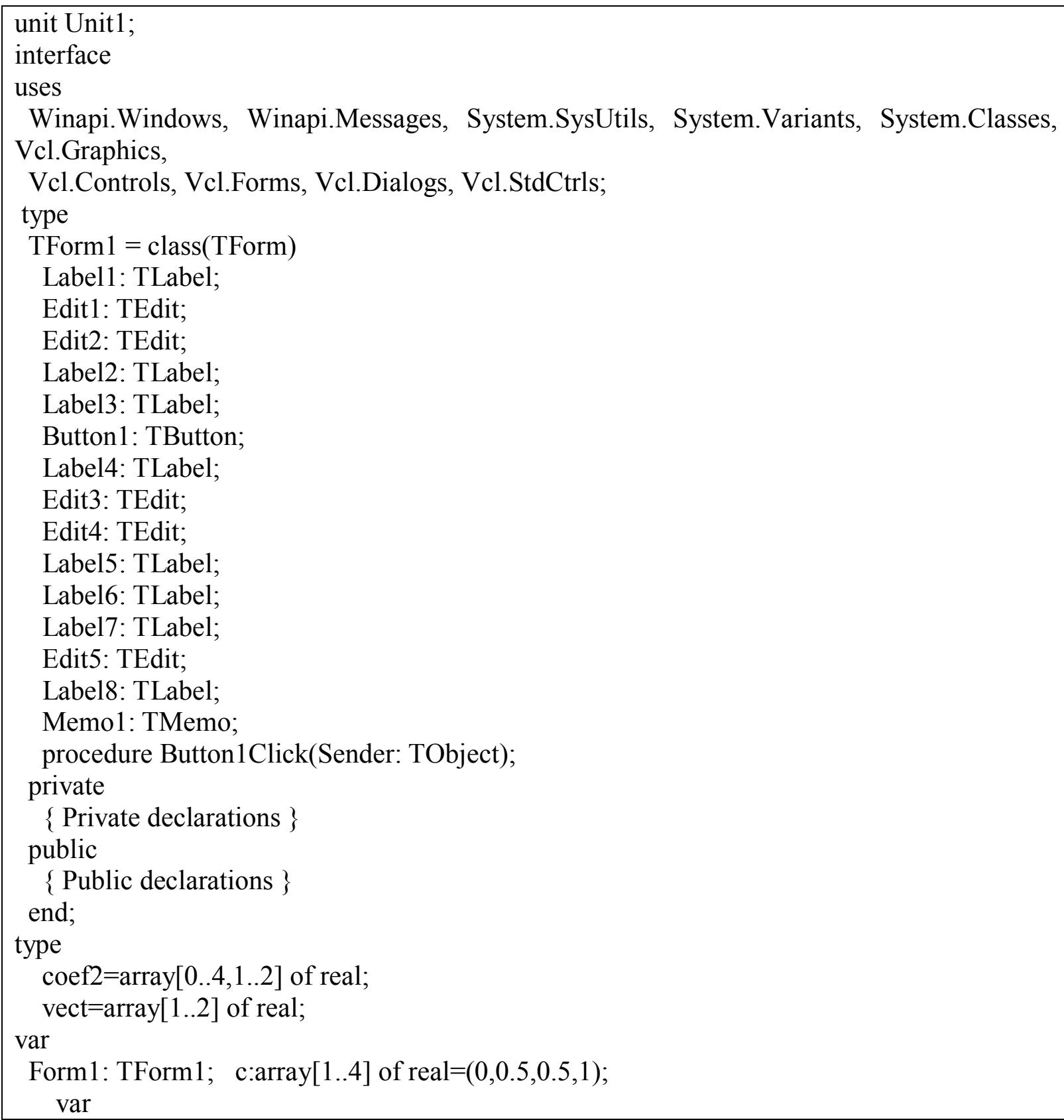




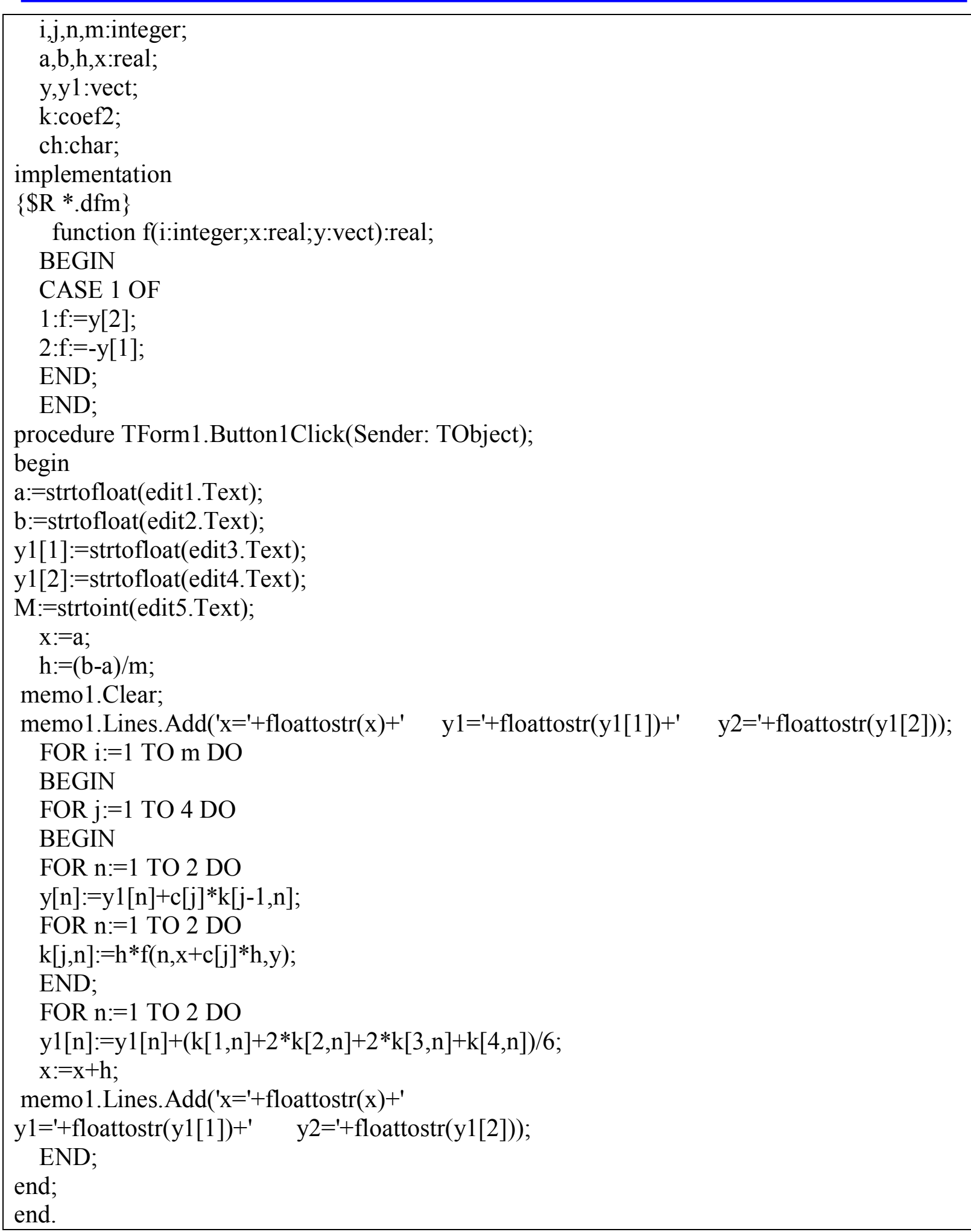




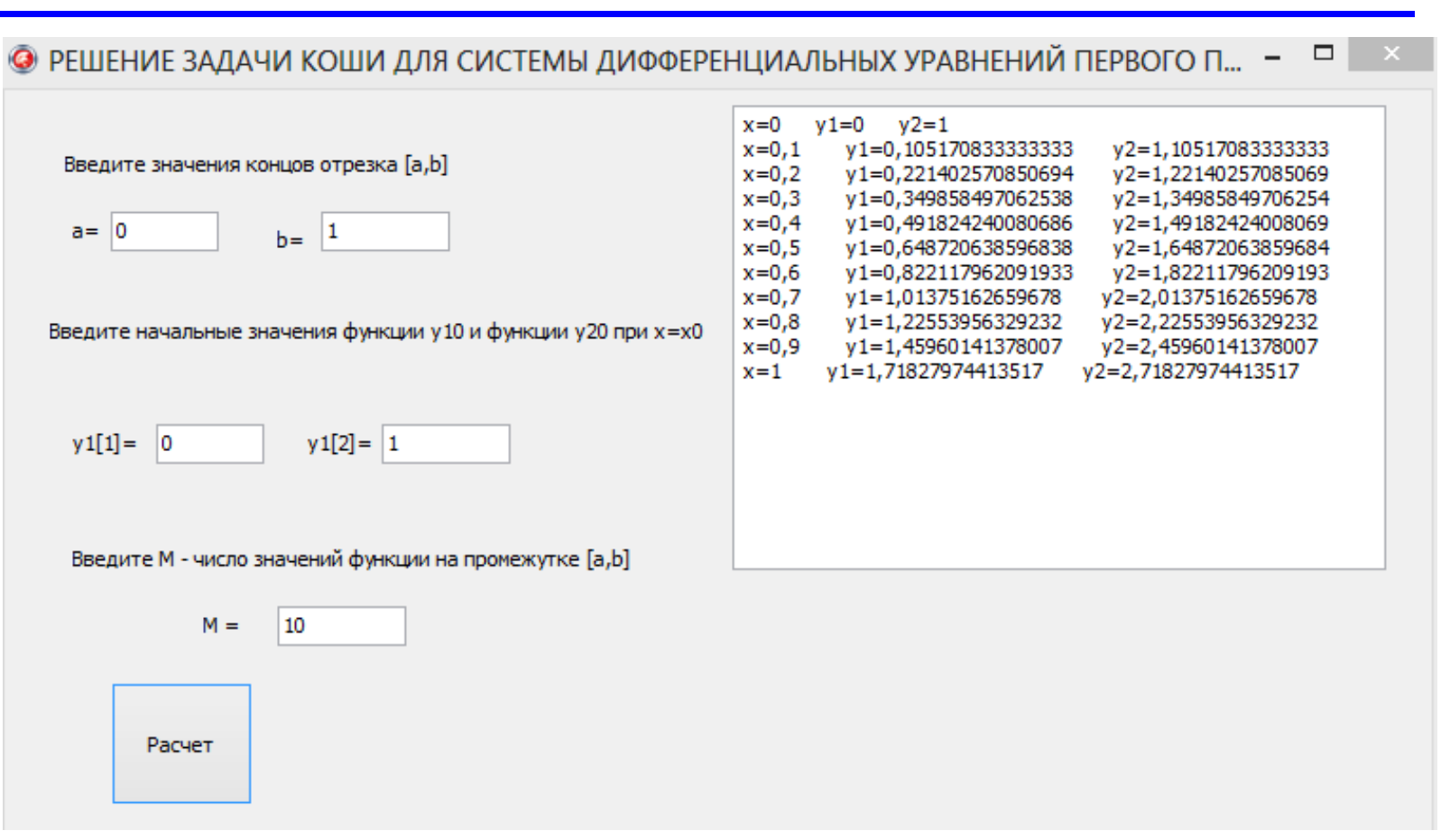

Рисунок 1 - Разработанная программа.

Описанные алгоритмы и разработанная программа (Рис.1) может быть использованна в различных исследованиях основанных на данном методе Рунге-Кутта, а также в процессе преподавания в университетах в качестве лабораторных работ.

\section{References:}

1. Пушкарь Е.А. Дифференциальные уравнения в задачах и примерах. М.:2007.-С.146

2. Шевцов А.Н. Математическое моделирование в прикладных задачах. Тараз 2012.C. $26-28$

3. Метод Рунге-Кутта. [Электронный ресурс] URL: http://otherreferats.allbest.ru/programming/00126107_0.html (дата доступа 25.04.2014)

4. ЧИСЛЕННОЕ РЕШЕНИЕ ОБЫКНОВЕННЫХ ДИФФЕРЕНЦИАЛЬНЫХ УРАВНЕНИЙ [Электронный ресурс] URL: http://kurs.ido.tpu.ru/courses/informat chem 2/modul 5.htm (дата доступа 25.04.2014)

5. Методы Рунге-Кутта [Электронный ресурс] URL: http://edu.dvgups.ru/METDOC/ENF/VMATEM/WM/METOD/VINOGRADOVA/WEB UMK/frame/8 1.htm (дата доступа 25.04.2014)

6. Метод Рунге-Кутта 4-го порядка для численного решения дифференциальных уравнений. [Электронный ресурс] URL: http://alexeypetrov.narod.ru/C/runge about.html (дата доступа 25.04.2014)

7. Метод Рунге-Кутта [Электронный ресурc] URL: http://www.simumath.net/library/book.html?code=Dif Ur method RK (дата доступа 25.04.2014)

8. Метод Рунге - Кутта [Электронный ресурс] URL: http://matica.org.ua/kursvisshey-matematiki-3/06-metod-runge-kutta (дата доступа 25.04.2014)

9. Численное решение задачи Коши для системы двух дифференциальных уравнений на языке Delphi [Электронный ресурс] URL:

http://knowledge.allbest.ru/mathematics/2c0a65625a3bc78b4d53a88421216d37 0.html (дата доступа 25.04.2014)

10. Численные методы [Электронный ресурс] URL: http://matica.org.ua/primeri/chislennie-metodi (дата доступа 25.04.2014) 
11. Как решить систему дифференциальных уравнений? [Электронный ресурс] URL: http://www.mathprofi.ru/sistemy differencialnyh uravnenij.html (дата доступа 25.04.2014)

12. РЕШЕНИЕ ОБЫКНОВЕННЫХ ДИФФЕРЕНЦИАЛЬНЫХ УРАВНЕНИЙ [Электронный ресурс] URL:

http://www.physchem.chimfak.rsu.ru/Source/NumMethods/ODE.html (дата доступа 25.04.2014)

13. О НЕКОТОРЫХ МЕТОДАХ ЧИСЛЕННОГО РЕШЕНИЯ ОБЫКНОВЕННЫХ ДИФФЕРЕНЦИАЛЬНЫХ УРАВНЕНИЙ ПЕРВОГО ПОРЯДКА С ПОСЛЕДУЮЩЕЙ РАЗРАБОТКОЙ ПРОГРАММЫ ДЛЯ ПК [ЭЛектроннЫЙ pecypc] URL: http://sibac.info/index.php/2009-07-01-10-21-16/6778-2013-03-11-0237-52 (дата доступа 25.04.2014)

14. ОБ УСТОЙЧИВОСТИ СИСТЕМ ЛИНЕЙНЫХ ДИФФЕРЕНЦИАЛЬНЫХ УРАВНЕНИЙ ВТОРОГО ТРЕТЬЕГО И ЧЕТВЕРТОГО ПОРЯДКА

[Электронный pecypc] URL: http:/www.science-education.ru/110-9669 (дата доступа 25.04.2014)

15. Численные методы решения обыкновенных дифференциальных уравнений и систем [Электронный ресурс] URL:

http://www.bibliofond.ru/view.aspx?id=539064 (дата доступа 25.04.2014) 